\title{
Relationship between day 1 and day 2 Vancomycin area under the curve values and emergence of heterogeneous Vancomycin-intermediate Staphylococcus aureus (hVISA) by Etest ${ }^{\oplus}$ macromethod among patients with MRSA bloodstream infections: a pilot study
}

Dmitriy M. Martirosov ${ }^{1}$, Monique R. Bidell ${ }^{1}$, Manjunath P. Pai ${ }^{1}$, Marc H. Scheetz ${ }^{2,3}$, Susan L. Rosenkranz ${ }^{4}$, Corey Faragon ${ }^{1}$, M. Malik', R. E. Mendes ${ }^{5}$, R. N. Jones ${ }^{5}$, Louise-Anne McNutt ${ }^{6}$ and Thomas P. Lodise ${ }^{1 *}$

\begin{abstract}
Background: In vitro data suggests that suboptimal initial vancomycin exposure may select for heterogeneous vancomycin-intermediate Staphylococcus aureus (hVISA) infections. However, no clinical studies have evaluated the relationship between initial vancomycin exposure and emergence of hVISA. This pilot study seeks to assess the relationship between day 1 and day 2 vancomycin area under the curve (AUC) and emergence of hVISA bloodstream infections (BSIs) by Etest ${ }^{\oplus}$ macromethod among patients with a non-hVISA BSI at baseline.

Methods: This was a retrospective cohort study of patients with methicillin-resistant Staphylococcus aureus (MRSA) BSIs at Albany Medical Center Hospital (AMCH) between January 2005 and June 2009. The vancomycin AUC exposure variables on day $1\left(\mathrm{AUC}_{0-24 \mathrm{~h}}\right)$ and day $2\left(\mathrm{AUC}_{24-48 \mathrm{~h}}\right)$ were estimated using the maximal a posteriori probability (MAP) procedure in ADAPT 5.

Results: There were 238 unique episodes of MRSA BSIs during the study period, 119 of which met inclusion criteria. Overall, hVISA emerged in 7/119 (5.9\%) of patients. All 7 cases of hVISA involved patients who did not achieve area under the curve over broth microdilution minimum inhibitory concentration $\left(A \cup C_{0-24 h} / M C_{B M D}\right.$ ) ratio of 521 or an $A U C_{24-48 h} / M_{I I C}$ BMD ratio of 650. No associations between other day 1 and day 2 AUC variables and emergence of hVISA were noted.

Conclusions: Although more data are needed to draw definitive conclusions, these findings suggest that hVISA emergence among patients with non-hVISA MRSA BSIs at baseline may be partially explained by suboptimal exposure to vancomycin in the first 1 to 2 days of therapy. At a minimum, these findings support further study of the relationship between initial vancomycin exposure and hVISA emergence among patients with MRSA BSIs in a wellpowered, multi-center, prospective trial.
\end{abstract}

Keywords: Vancomycin, Heteroresistance, Staphylococcus aureus, MRSA

\footnotetext{
* Correspondence: thomas.lodise@acphs.edu

'Albany College of Pharmacy and Health Sciences, Albany, NY 12208-3492,

USA

Full list of author information is available at the end of the article
} 


\section{Background}

Vancomycin is often employed as a first-line treatment of serious infections due to methicillin-resistant Staphylococcus aureus (MRSA) [1]. While susceptibility to vancomycin among MRSA remains near $100 \%$, there are growing report of vancomycin-susceptible strains that exhibit a "heteroresistant" phenotype. Heterogeneous vancomycin-intermediate methicillin-resistant $S$. aureus (hVISA) is defined by the presence of an intermediateresistant subpopulation at a frequency of $10^{-5}-10^{-6} \mathrm{CFU}$ in a susceptible isolate with an $\mathrm{MIC}_{\mathrm{BMD}} \leq 2 \mathrm{mg} / \mathrm{L}$ [2]. Laboratory studies suggest that suboptimal vancomycin exposure early in the course of therapy may select for hVISA during or shortly after completion of therapy [3]. This in vitro finding is concerning as data suggest that patients with hVISA infections are less responsive to vancomycin [4]. Despite this potential association between suboptimal exposure to vancomycin and hVISA emergence, clinical studies evaluating the relationship between initial vancomycin exposure and emergence of hVISA are largely nonexistent. This pilot study seeks to assess the relationship between day 1 and day 2 vancomycin area under the curve (AUC) and emergence of hVISA bloodstream infections (BSIs) by Etest ${ }^{\oplus}$ macromethod among patients with a nonhVISA BSI at baseline. Bayesian techniques were used to estimate the vancomycin concentration-time profile for each patient and the relationships between area under the curve (AUC), AUC/MIC, minimum concentration (Cmin), and AUC/MIC and hVISA emergence were examined. The Bayesian approach used to estimate exposure profiles in this study has recently been validated as a method to estimate vancomycin exposure values with low bias and high precision in situations where trough-only PK data are available.

\section{Methods}

This was a retrospective cohort study of patients with MRSA BSIs at Albany Medical Center Hospital (AMCH) between January 2005 and June 2009 performed in accordance with the AMCH Institutional Review Board. The study was approved by expedited review by the Institutional Review Board of AMCH and a HIPAA waiver was obtained. Patients were included if they met the following criteria: (1) age $\geq 18$ years; (2) absolute neutrophil count $\geq 1000$ cells $/ \mathrm{mm}^{3}$; (3) not receiving renal replacement therapy; (4) MRSA blood culture met the Centers for Disease Control and Prevention (CDC) criteria for BSI [5]; (5) index and at least 1 subsequent MRSA bloodstream isolates were available for phenotypic characterization; (6) index MRSA isolate was negative for hVISA by Etest ${ }^{\circ}$ macromethod [6]; (7) received vancomycin within $48 \mathrm{~h}$ of index blood culture; (8) received at least 2 days of vancomycin; and (9) had at least one vancomycin level collected within 5 days of starting treatment.
The study methods are comparable to our recently published study [7]. Isolates were identified as S. aureus according to standard methods. Initial susceptibility testing for oxacillin resistance was performed according to CLSI guidelines [8]. Isolates were stored in trypticase soy broth with $20 \%$ glycerol at $-70{ }^{\circ} \mathrm{C}$. All MRSA bloodstream isolates obtained from the patient were shipped to JMI Laboratories (North Liberty, IA) for hVISA screening and determination of MIC through broth microdilution method (BMD) and Etest ${ }^{\bullet}$ strips (lot\# BJ0469) according to the manufacturer's instructions (bioMérieux, Marcy l'Etoile, France). BMD and Etest ${ }^{\circ}$ MICs were performed for MRSA using S. aureus ATCC 29213 and Enterococcus faecalis ATCC 29212 as quality control strains. Screening for hVISA was performed by Etest $^{\oplus}$ macromethod using $\mathrm{Mu} 3$ as a control strain as described by Wootton et al. [6].

All inpatient antibiotic treatment and vancomycin concentration data during the study period were collected. The vancomycin area under the curve exposure variables on day $1\left(\mathrm{AUC}_{0-24 \mathrm{~h}}\right)$ and day $2\left(\mathrm{AUC}_{24-48 \mathrm{~h}}\right)$ were estimated using the maximal a posteriori probability (MAP) procedure in ADAPT 5 as previously described $[7,9,10]$. This method has been validated to estimate AUC values with low bias and high precision utilizing trough-only sampling [10]. With the Bayesian posterior PK information for a given individual, ADAPT 5 was also used to estimate $\mathrm{Cmin}_{24 \mathrm{~h}}$ and $\mathrm{Cmin}_{48 \mathrm{~h}}$. The primary vancomycin exposure variables considered in the analyses included: (1) $\mathrm{AUC}_{0-24 \mathrm{~h}}$; (2) $\mathrm{AUC}_{0-24 \mathrm{~h}}$ / $\mathrm{MIC}_{\mathrm{BMD}}$; (3) $\mathrm{AUC}_{0-24 \mathrm{~h}} / \mathrm{MIC}_{\mathrm{Etest}} ;$ (4) $\mathrm{AUC}_{24-48 \mathrm{~h}}$; (5) $\mathrm{AUC}_{24-48 \mathrm{~h}} / \mathrm{MIC}_{\mathrm{BMD}}$; (6) $\mathrm{AUC}_{24-48 \mathrm{~h}} / \mathrm{MIC}_{\text {Etest }}$; (7) $\mathrm{C}_{\text {min24h }}$; and (8) $\mathrm{Cmin}_{48 \mathrm{~h}}$. We assessed both $\mathrm{AUC}_{0-24 \mathrm{~h}}$ and $\mathrm{AUC}_{24-48 \mathrm{~h}}$, as $\mathrm{AUC}_{0-24 \mathrm{~h}}$ reflects initial exposure conditions and is consistent with the exposure profile evaluated in animal model studies $[11,12]$, while $\mathrm{AUC}_{24-48 \mathrm{~h}}$ represents a near steady-state exposure profile associated with a maintenance vancomycin regimen.

The primary outcome of the study was emergence of hVISA, defined as the documentation of a hVISA bloodstream isolate after $24 \mathrm{~h}$ of vancomycin treatment and up to 60 days after completion of therapy among patients with a non-hVISA BSI at baseline. Classification and Regression Tree (CART) analysis was used to identify vancomycin day 1 and day 2 AUC exposure variables associated with an increased probability of hVISA emergence. Bivariate comparisons were assessed using Fisher's exact test, Student's t test, and Mann-Whitney $U$ test. Poisson regression with robust variance estimates analyses were performed to identify variables independently associated with hVISA emergence. Each CARTderived vancomycin AUC exposure variable was evaluated in a separate regression analysis. All baseline variables associated with emergence of hVISA at a $P$ - 
value $<0.2$ were considered at model entry and a stepwise approach was employed to identify independent variables. Calculations were performed using SAS version 9.3 (Cary, North Carolina) and CART software (Salford Systems, San Diego, California).

\section{Results}

There were 238 unique episodes of MRSA BSIs during the study period, 119 of which met inclusion criteria. Emergence of hVISA by Etest ${ }^{\circ}$ macromethod was observed in 7 (5.9\%) patients. Nearly all hVISA isolates emerged during vancomycin treatment and 4 of 7 appeared within 15 days of the index MRSA blood culture (Table 1). One case of hVISA emerged after therapy discontinuation. There was a documented intervention in the medical record related to source control in $>95 \%$ of cases when warranted. In the CART analysis, patients failing to achieve an $\mathrm{AUC}_{0-24 \mathrm{~h}} / \mathrm{MIC}_{\mathrm{BMD}}$ ratio of 521 and an $\mathrm{AUC}_{24-48 \mathrm{~h}} / \mathrm{MIC}_{\mathrm{BMD}}$ ratio of 650 had increased incidences of hVISA emergence. Among the 61 patients who did not achieve an $\mathrm{AUC}_{0-24 \mathrm{~h}} / \mathrm{MIC}_{\mathrm{BMD}}$ ratio of 521 or an $\mathrm{AUC}_{24-48 \mathrm{~h}} / \mathrm{MIC}_{\mathrm{BMD}}$ ratio of $650,11.5 \%$ had emergence of hVISA. All 7 cases of hVISA emerged in patients who did not achieve $\mathrm{AUC}_{0-24 \mathrm{~h}} / \mathrm{MIC}_{\mathrm{BMD}}$ ratio of 521 or an $\mathrm{AUC}_{24-48 \mathrm{~h}} / \mathrm{MIC}_{\mathrm{BMD}}$ ratio of 650 ; variables were not completely mutually exclusive (Table 1 ). No associations between other day 1 and day 2 AUC variables and emergence of hVISA were noted (data not shown). No association between $\mathrm{Cmin}_{24 \mathrm{~h}}$ and $\mathrm{Cmin}_{48 \mathrm{~h}}$ was noted. Among patients with hVISA, the mean (SD) $\mathrm{Cmin}_{24 \mathrm{~h}}$ and $\mathrm{Cmin}_{48 \mathrm{~h}}$ were 7.3 (3.4) and 13.4 (5.1), respectively. Similar mean (SD) $\mathrm{Cmin}_{24 \mathrm{~h}}$ and $\mathrm{Cmin}_{48 \mathrm{~h}}$ were observed among non-hVISA patients (8.7 (4.8) and 11.3 (6.0), respectively).

Comparisons between baseline characteristics and emergence of hVISA and day 1 and day 2 AUC/MIC BMD ratio groups and hVISA emergence are shown in Table 2. Compared to their non-hVISA counterparts, hVISA patients were entirely male, had lower baseline mean renal function, and were more likely to have infective endocarditis and receiving immunosuppressive therapy. Relative to patients with an $\mathrm{AUC}_{0-24 \mathrm{~h}} / \mathrm{MIC}_{\mathrm{BMD}}$ ratio $<521$, patients with an $\mathrm{AUC}_{0-24 \mathrm{~h}} / \mathrm{MIC}_{\mathrm{BMD}}$ ratio of $\geq 521$ were significantly more likely to be older and have heart failure, but less likely to have a $\mathrm{MIC}_{\mathrm{BMD}} \geq 1 \mathrm{mg} / \mathrm{L}$. Patients with an $\mathrm{AUC}_{24-48 \mathrm{~h}} / \mathrm{MIC}_{\mathrm{BMD}}$ ratio $\geq 650$ were more likely to have recent surgery in the previous 30 days and less likely to have $\mathrm{MIC}_{\mathrm{BMD}} \geq 1 \mathrm{mg} / \mathrm{L}$ relative to those with an $\mathrm{AUC}_{24-48 \mathrm{~h}} / \mathrm{MIC}_{\mathrm{BMD}}$ ratio $<650$.

Results of the Poisson regression analyses are shown in Table 3. When controlling for all baseline variables associated with emergence of hVISA at a $P$-value $<0.2$, patients with a $\mathrm{AUC}_{0-24 \mathrm{~h}} / \mathrm{MIC}_{\mathrm{BMD}} \geq 521$ had a lower risk of hVISA emergence (relative risk $(\mathrm{RR})=0.14,95 \%$ confidence interval (CI): 0.03-0.60) in the day 1 model. Presence of infective endocarditis, $\mathrm{CL}_{\mathrm{CR}}$, and skin and soft tissue source of infection were also found to be independently associated with hVISA emergence. In the day 2 model, $\mathrm{AUC}_{24-48 \mathrm{~h}} / \mathrm{MIC}_{\mathrm{BMD}}$ ratio $\geq 650$ was found to be associated with lower risk of hVISA emergence but the associated $p$-value was 0.08 ( $R=0.16,95 \%$ CI: $0.02-$ $1.28)$. Other variables associated with hVISA emergence in the day 2 model included $\mathrm{CL}_{\mathrm{CR}}$ and presence of infective endocarditis.

\section{Discussion}

This pilot study evaluated the relationship between vancomycin exposure and emergence of hVISA by Etest ${ }^{\circ}$ macromethod in patients with non-hVISA MRSA BSIs at baseline. Nearly all 7 cases of hVISA emerged during vancomycin treatment and 4 of 7 appeared within 15 days of the index MRSA blood culture (Table 1). One case of hVISA emerged after therapy discontinuation. Using a validated Bayesian technique to estimate each patient's $\mathrm{AUC}_{0-24 \mathrm{~h}}$ and $\mathrm{AUC}_{24-48 \mathrm{~h}}$ values with limited $\mathrm{PK}$ sampling [10], we found that failure to achieve an $\mathrm{AUC}_{0-}$ ${ }_{24 \mathrm{~h}} / \mathrm{MIC}_{\mathrm{BMD}}$ ratio of 521 and an $\mathrm{AUC}_{24-48 \mathrm{~h}} / \mathrm{MIC}_{\mathrm{BMD}}$ ratio of 650 was associated with an increased risk of hVISA emergence. Of the 7 patients for which hVISA emerged, 6 occurred in patients with $\mathrm{AUC}_{0-24 \mathrm{~h}} / \mathrm{MIC}_{\mathrm{BMD}}$ ratio less than 521 and $\mathrm{AUC}_{24-48 \mathrm{~h}} / \mathrm{MIC}_{\mathrm{BMD}}$ ratio less than 650. Interestingly, all 7 cases of hVISA emerged in

Table 1 Clinical characteristics of patients in whom hVISA population was detected

\begin{tabular}{|c|c|c|c|c|c|c|c|}
\hline & \multicolumn{7}{|c|}{ Patient Number } \\
\hline & 1 & 2 & 3 & 4 & 5 & 6 & 7 \\
\hline $\mathrm{AUCO}_{0-24 \mathrm{~h}} / \mathrm{MIC}_{\mathrm{BMD}}$ ratio & 420.8 & 226.4 & 358.6 & 519.5 & 251.9 & 302.6 & 759.6 \\
\hline$A \cup C_{24-48 h} / M C_{B M D}$ ratio & 506.6 & 600.9 & 505.6 & 806.6 & 534.7 & 442.1 & 545.3 \\
\hline Creatinine clearance (mL/min) & 34.2 & 22.8 & 34.5 & 22.1 & 65.7 & 25.3 & 8.4 \\
\hline Days from index MRSA blood culture to emergence of hVISA & 62 & 2 & 2 & 14 & 15 & 62 & 23 \\
\hline Days from start of vancomycin to emergence of hVISA & 62 & 2 & 1 & 14 & 13 & 61 & 22 \\
\hline hVISA emergence on treatment & Y & Y & $\mathrm{Y}$ & $\mathrm{Y}$ & Y & $\mathrm{N}$ & Y \\
\hline
\end{tabular}

Abbreviations: $h$ VISA heterogeneous vancomycin-intermediate Staphylococcus aureus, $A U C_{0-24 h} / M_{I} C_{B M D}$ area under the curve from 0 to $24 \mathrm{~h}$ over broth microdilution minimum inhibitory concentration, $A \cup C_{24-48 h} / M I C_{B M D}$, area under the curve from 24 to 48 h over broth microdilution minimum inhibitory concentration, $Y$ yes, $N$, no 
Table 2 Associations between baseline characteristics and (a) CART-derived $A U C_{0-24 h} / M C_{B M D}$ and $A U C_{24-48 h} / M C_{B M D}$ groups and (b) emergence of hVISA

\begin{tabular}{|c|c|c|c|c|c|c|}
\hline & $\begin{array}{l}\mathrm{AUC}_{0-24 \mathrm{~h}} / \mathrm{MIC}_{\mathrm{BMD}} \\
<521 \\
(\mathrm{~N}=52)\end{array}$ & $\begin{array}{l}\mathrm{AUC}_{24-48 \mathrm{~h}} / \mathrm{MIC}_{\mathrm{BMD}} \\
\geq 521 \\
(\mathrm{~N}=67)\end{array}$ & $\begin{array}{l}\mathrm{AUC}_{24-48 h} / \mathrm{MIC}_{\mathrm{BMD}} \\
<650 \\
(\mathrm{~N}=54)\end{array}$ & $\begin{array}{l}\mathrm{AUC}_{24-48 \mathrm{~h}} / \mathrm{MIC}_{\mathrm{BMD}} \\
\geq 650 \\
(\mathrm{~N}=65)\end{array}$ & $\begin{array}{l}\text { Non-hVISA } \\
(N=112)\end{array}$ & $\begin{array}{l}\text { hVISA } \\
(N=7)\end{array}$ \\
\hline Age in years, mean (SD) & $57.1(15.6)^{* *}$ & $64.5(14.6)^{* *}$ & $58.0(17.1)^{*}$ & $64.0(14.4)^{*}$ & $60.8(15.6)$ & $68.4(10.9)$ \\
\hline Sex-male & $34(65.4)$ & $49(73.1)$ & $39(72.2)$ & $44(67.7)$ & $76(67.9) 1^{*}$ & $7(100)^{*}$ \\
\hline Weight in kg, mean (SD) & $86.5(28.3)$ & $82.6(31.7)$ & $84.7(27.9)$ & $84.4(33.0)$ & $84.0(30.7)$ & $89.5(20.5)$ \\
\hline $\begin{array}{l}\text { Recent residence in healthcare institution } \\
\text { in previous } 6 \text { months }\end{array}$ & $30(57.5)$ & $43(64.2)$ & $27(50.0)$ & $40(61.5)$ & $68(60.7)$ & $5(71.4)$ \\
\hline Diabetes mellitus & 19 (36.5) & $33(49.3)^{*}$ & $21(38.9)$ & $31(47.7)$ & $48(42.9)$ & $4(57.1)$ \\
\hline Heart failure & $11(21.2)$ & $27(40.3)^{* *}$ & $13(24.1)^{*}$ & $25(38.5)^{*}$ & $37(33.0)$ & $1(2.6)$ \\
\hline COPD & $12(23.1)$ & $26(38.8)^{*}$ & $17(31.5)$ & $21(32.3)$ & $35(31.3)$ & $3(42.9)$ \\
\hline Hepatic dysfunction & $1(1.9)$ & $4(6.0)$ & $1(1.9)$ & $4(6.2)$ & $5(4.5)$ & $0(0.0)$ \\
\hline Malignancy & $19(36.5)$ & $14(20.9)^{*}$ & $17(31.5)$ & $16(24.6)$ & $30(26.8)$ & $3(42.9)$ \\
\hline Decubitus ulcers & $11(21.2)$ & $14(20.9)$ & $12(22.2)$ & $13(20.0)$ & $24(21.4)$ & $1(14.3)$ \\
\hline Immunosuppressants & $13(25.0)$ & $10(14.9)$ & $12(22.2)$ & $11(16.9)$ & $20(17.9)^{*}$ & $3(42.9)^{*}$ \\
\hline HIV & $3(5.8)$ & $1(1.5)$ & $3(5.6)$ & $1(1.5)$ & $4(3.6)$ & $0(0.0)$ \\
\hline History of cerebrovascular event & $5(9.6)$ & $8(11.9)$ & $5(9.3)$ & $8(12.3)$ & $13(11.6)$ & $0(0.0)$ \\
\hline Recent surgery in previous 30 days & $21(40.4)$ & $19(28.4)^{*}$ & $26(48.1)^{* *}$ & $14(21.5)^{* *}$ & $36(32.1)$ & $4(57.1)$ \\
\hline Recent antibiotics in previous 30 days & $29(55.8)$ & $36(53.7)$ & $29(53.7)$ & $36(55.4)$ & $62(55.4)$ & $3(42.9)$ \\
\hline Prior vancomycin in past 30 days & $11(21.2)$ & $9(13.4)$ & $10(18.5)$ & $10(15.4)$ & $20(17.9)$ & $0(0.0)$ \\
\hline $\begin{array}{l}\text { Length of stay in days prior to index culture } \\
\text { collection, median (IQR) days }\end{array}$ & $2(0-14)$ & $1(0-12)$ & $3(0-19)$ & $1(0-13)$ & $3(0-14)^{*}$ & $0(0-0)^{*}$ \\
\hline Intensive care unit at onset & $15(28.8)$ & $26(38.8)$ & $18(33.3)$ & $23(35.4)$ & $40(35.7)$ & $1(14.3)$ \\
\hline Baseline $\mathrm{CL}_{\mathrm{CR}}$ in $\mathrm{mL} /$ min, mean (SD) & $77.8(56.8)$ & $71.1(46.9)$ & $80.1(58.4)$ & $69.8(47.0)$ & $76.7(51.5)^{* *}$ & $30.4(17.8)^{* *}$ \\
\hline APACHE-\|l Score, mean (SD) & $13.3(5.6)$ & $14.4(6.5)$ & $14.2(6.2)$ & $14.1(6.0)$ & $13.7(6.1)^{*}$ & $17.7(4.5)^{*}$ \\
\hline $\mathrm{MIC}_{\mathrm{BMD}} \geq 1 \mathrm{mg} / \mathrm{L}$ & $16(30.8)^{* *}$ & $8(9.0)^{* *}$ & $16(29.6)^{* *}$ & $6(9.2)^{* *}$ & $18(16.1)^{* *}$ & $4(57.1)^{* *}$ \\
\hline $\mathrm{MIC}_{\text {Etest }} \geq 1.5 \mathrm{mg} / \mathrm{L}$ & $35(67.3)^{*}$ & $35(52.2)^{*}$ & $35(64.8)$ & $35(53.8)$ & $65(58.0)$ & $5(71.4)$ \\
\hline \multicolumn{7}{|l|}{ Source of bloodstream infection } \\
\hline - Intravenous catheter & $25(48.1)^{*}$ & $24(35.8)^{*}$ & $23(42.6)$ & $26(40.0)$ & $48(42.9)$ & $1(14.3)$ \\
\hline - Skin and soft tissue & $13(25.0)$ & $14(20.9)$ & $15(27.8)$ & $12(18.5)$ & $23(20.5)^{* *}$ & $4(57.1)^{* *}$ \\
\hline - Bone and joint & $2(3.8)$ & $6(9.0)$ & $2(3.7)$ & $6(9.2)$ & $8(7.1)$ & $0(0.0)$ \\
\hline - Respiratory tract & $2(3.8)$ & $7(10.4)$ & $2(3.7)^{*}$ & $7(10.8)^{*}$ & $9(8.0)$ & $0(0.0)$ \\
\hline • Intra-abdominal & $3(5.8)$ & $4(6.0)$ & $4(7.4)$ & $3(4.6)$ & $6(5.4)$ & $1(14.3)$ \\
\hline - Urinary tract & $1(1.9)$ & $1(1.5)$ & $1(1.9)$ & $1(1.5)$ & $2(1.8)$ & $0(0.0)$ \\
\hline - Infected graft/device & $2(3.8)$ & $5(7.5)$ & $2(3.7)$ & $5(7.7)$ & $5(5.4)$ & $1(14.3)$ \\
\hline - Unknown & $4(7.7)$ & $6(9.0)$ & $5(9.3)$ & $5(7.7)$ & $10(8.9)$ & $0(0.0)$ \\
\hline Presence of Infective Endocarditis & $9(17.3)$ & $8(11.9)$ & $9(16.7)$ & $8(12.3)$ & $14(12.6)^{*}$ & $3(42.9)^{*}$ \\
\hline
\end{tabular}

All data presented as number (percent) unless otherwise indicated

${ }^{*}$-value $<0.2$

**P-value $<0.05$

Abbreviations: $h$ VISA heterogeneous vancomycin-intermediate Staphylococcus aureus, SD standard deviation, COPD chronic obstructive pulmonary disease, HIV human immunodeficiency virus $I Q R$ interquartile range, $C L_{C R}$ creatinine clearance, $A P A C H E-I I$ Acute Physiology and Chronic Health Evaluation II, $M I C_{B M D}$ broth microdilution minimum inhibitory concentration, $M I C_{\text {Etest }}$ Etest ${ }^{\oplus}$ minimum inhibitory concentration

patients who did not achieve $\mathrm{AUC}_{0-24 \mathrm{~h}} / \mathrm{MIC}_{\mathrm{BMD}}$ ratio of 521 or an $\mathrm{AUC}_{24-48 \mathrm{~h}} / \mathrm{MIC}_{\mathrm{BMD}}$ ratio of 650 (Table 1), suggesting the dual importance of both day 1 and day 2 vancomycin AUC exposures and cumulative vancomycin exposure over the first 2 days of therapy. Presence of infective endocarditis was also found to be associated with an increased risk of hVISA emergence in the multivariate analyses. This is consistent with a meta-analysis from van Hal and Paterson [13] which described an association between high-inoculum infections (e.g. 
Table 3 Variables associated with emergence of hVISA in the multivariate analyses

\begin{tabular}{lllll}
\hline \multicolumn{1}{c}{ Variable } & $\begin{array}{l}\text { Relative } \\
\text { Risk }\end{array}$ & $\begin{array}{l}\text { 95\% confidence } \\
\text { interval }\end{array}$ & P-value \\
\hline Day 1 & $\mathrm{AUC}_{0-24 h} / \mathrm{MIC}_{\mathrm{BMD}} \geq 521$ & 0.14 & $0.03-0.60$ & 0.008 \\
& $\mathrm{CL}_{\mathrm{CR}}$ & 0.93 & $0.88-0.98$ & 0.004 \\
& Presence of IE & 4.94 & $1.67-14.68$ & 0.004 \\
& Skin and soft tissue source & 4.89 & $1.43-16.71$ & 0.01 \\
Day 2 & $\mathrm{AUC}_{24-48 \mathrm{~h}} / \mathrm{MIC}_{\mathrm{BMD}} \geq 650$ & 0.16 & $0.02-1.28$ & 0.08 \\
& $\mathrm{CL}_{\mathrm{CR}}$ & 0.95 & $0.91-0.98$ & 0.007 \\
& Presence of IE & 4.62 & $1.67-12.77$ & 0.003 \\
\hline
\end{tabular}

Abbreviations: $h$ VISA heterogeneous vancomycin-intermediate Staphylococcus aureus, $A \cup C_{0-24 h} / M I C_{B M D}$ area under the curve from 0 to $24 \mathrm{~h}$ over broth microdilution minimum inhibitory concentration, $A \cup C_{24-48 h} / M I C_{B M D}$ area under the curve from $24-48 \mathrm{~h}$ over broth microdilution minimum inhibitory concentration, IE infective endocarditis

infective endocarditis) and hVISA. Future studies should aim to better elucidate these findings, including the possibility that an alternative exposure threshold may exist for these patients. The observed associations between decreased baseline creatinine clearance and hVISA infection in the multivariate analyses are also not surprising given the number of reports of resistance emergence in this population throughout the literature [14].

Several things should be considered when interpreting these results. This study is subject to limitations inherent to the study design, namely confounding and selection bias. However, the comparable distribution of baseline characteristics between the CART-derived $\mathrm{AUC}_{24-48 \mathrm{~h}} / \mathrm{MIC}_{\mathrm{BMD}}$ groups and persistence of findings in the multivariate analysis suggest that confounding is unlikely to contribute to the exposure-response observed findings. Results may not be applicable to neutropenic and dialysis patients as these patients were excluded from this study; AMCH does not have an appreciable neutropenic population, and exposure variables are numerous and difficult to characterize for hemodialysis-dependent patients (e.g. sporadic dosing, inconsistent removal volumes due to variable durations and filters). Both of these excluded populations are frequently identified at risk for resistance emergence [14] and the incidence of hVISA emergence may be higher in these populations than that observed in our study population. Two study patients had emergence of hVISA within 2 days of vancomycin therapy. It is unclear if suboptimal exposure to vancomycin selected for these isolates or if they were present in a sufficient density for detection at baseline. Clear delineation between exposure accrual and subsequent resistance emergence should be an important consideration in future studies as well.

Our CART-derived $\mathrm{AUC}_{\mathrm{h}} / \mathrm{MIC}_{\mathrm{BMD}}$ ratio breakpoints for defining hVISA emergence can only be considered a rough estimation due to the small sample size. While vancomycin dose and concentration collections used to generate estimates of vancomycin PK parameters for each individual patient occurred within the first 5 days of therapy, most were obtained on days 1-3. Limiting collection to days 1 and 2 to estimate $\mathrm{AUC}_{0-24 \mathrm{~h}}$ and $\mathrm{AUC}_{24-48 \mathrm{~h}}$ would have been preferred, but this would have severely restricted our already small study sample. For this reason, we employed a vancomycin population PK model as the Bayesian prior that included creatinine clearance as a covariate to account for potential changes in renal function during the first few days of therapy. As previously described, use of a vancomycin population PK model including creatinine clearance ensured a good fit of the model to the observed vancomycin concentrations collected over the first few days of therapy relative to predicted concentrations in our study population [7]. Future studies should consider intensive PK collections on the day the exposure profile is estimated.

Lastly, hVISA phenotypes were not confirmed by population analysis profile (PAP)-area under the curve (AUC). However, the macrodilution Etest ${ }^{\circ}$ used in the study has been shown to be a viable screening method with a specificity ranging from $89-98 \%$ with few false positives [6]. The presence of hVISA by macrodilution Etest $^{\oplus}$ has also shown to be predictive of patient outcomes across of a number of studies, further highlighting the importance of findings from our pilot investigation and need for further study [13]. Finally, future hypothesis analyzing studies should include DNA fingerprinting of the first isolate and subsequent isolates to ensure that isolates are the same.

\section{Conclusion}

In conclusion, the findings from this pilot suggest that in patients with non-hVISA MRSA BSIs at baseline, failure to achieve an $\mathrm{AUC}_{0-24 \mathrm{~h}} / \mathrm{MIC}_{\mathrm{BMD}}$ ratio of 521 or an $\mathrm{AUC}_{24-48 \mathrm{~h}} / \mathrm{MIC}_{\mathrm{BMD}}$ ratio of 650 is associated with hVISA emergence by macrodilution Etest ${ }^{\circ}$. Though we acknowledge that many factors contribute to emergence of hVISA, and that the occurrence of all outcomes are multi-factorial, our pilot findings strongly suggest that day 1 and day 2 vancomycin AUC profiles may be contributory. Definitive conclusions are limited by the retrospective and exploratory nature of the study and its small sample size. However, these data contribute to existing literature in support of AUC monitoring during the first $48 \mathrm{~h}$ of vancomycin therapy in patients with MRSA BSIs [7]. Prospective validation in a larger-scale, multi-center clinical trial is necessary to determine the vancomycin pharmacokinetic/pharmacodynamic profile associated with hVISA emergence given its potentially critical importance for defining early optimal vancomycin therapy. 


\section{Abbreviations}

AMCH: Albany Medical Center Hospital; AUC: Area under the curve; AUC $_{0}$

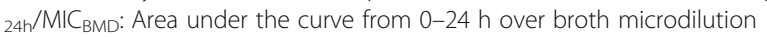
minimum inhibitory concentration; $\mathrm{AUC}_{24-48 h} / \mathrm{MIC}_{\mathrm{BMD}}$ : Area under the curve from 24-48 h over broth microdilution minimum inhibitory concentration; BSIs: Bloodstream infections; CART: Classification and Regression Tree; hVISA: Heterogeneous vancomycin-intermediate Staphylococcus aureus; IE: Infective endocarditis; MAP: Maximal a posteriori; MRSA: Methicillin resistant Staphylococcus aureus

\section{Acknowledgements}

None.

\section{Funding}

This work was supported in part by the National Institute of Allergy and Infectious Diseases (grant number Al105742) to (TPL and MHS). Its contents are solely the responsibility of the authors and do not necessarily represent the official views of the NIAID.

\section{Availability of data and materials}

Restrictions apply to the availability of these data, which were used under license for the current study, and so are not publicly available. Data are, however, available from the authors upon reasonable request.

\section{Authors' contributions}

DMM substantially contributed to the conception and design of the study, acquisition and analysis of data and interpretation of results, writing and critical review of the manuscript, gave approval of the final version of the manuscript to be published, and agreed to be accountable for the integrity of the data and the accuracy of the work. MRB substantially contributed to the conception and design of the study, acquisition and analysis of data and interpretation of results, writing and critical review of the manuscript, gave approval of the final version of the manuscript to be published, and agreed to be accountable for the integrity of the data and the accuracy of the work. MPP substantially contributed to the conception and design of the study, acquisition and analysis of data and interpretation of results, writing and critical review of the manuscript, gave approval of the final version of the manuscript to be published, and agreed to be accountable for the integrity of the data and the accuracy of the work. MHS substantially contributed to the conception and design of the study, acquisition and analysis of data and interpretation of results, writing and critical review of the manuscript, gave approval of the final version of the manuscript to be published, and agreed to be accountable for the integrity of the data and the accuracy of the work. SLR substantially contributed to the conception and design of the study, acquisition and analysis of data and interpretation of results, writing and critical review of the manuscript, gave approval of the final version of the manuscript to be published, and agreed to be accountable for the integrity of the data and the accuracy of the work. CF substantially contributed to the conception and design of the study, acquisition and analysis of data and interpretation of results, writing and critical review of the manuscript, gave approval of the final version of the manuscript to be published, and agreed to be accountable for the integrity of the data and the accuracy of the work. MM substantially contributed to the conception and design of the study, acquisition and analysis of data and interpretation of results, writing and critical review of the manuscript, gave approval of the final version of the manuscript to be published, and agreed to be accountable for the integrity of the data and the accuracy of the work. REM substantially contributed to the conception and design of the study, acquisition and analysis of data and interpretation of results, writing and critical review of the manuscript, gave approval of the final version of the manuscript to be published, and agreed to be accountable for the integrity of the data and the accuracy of the work. RNJ substantially contributed to the conception and design of the study, acquisition and analysis of data and interpretation of results, writing and critical review of the manuscript, gave approval of the final version of the manuscript to be published, and agreed to be accountable for the integrity of the data and the accuracy of the work. LM substantially contributed to the conception and design of the study, acquisition and analysis of data and interpretation of results, writing and critical review of the manuscript, gave approval of the final version of the manuscript to be published, and agreed to be accountable for the integrity of the data and the accuracy of the work. TPL substantially contributed to the conception and design of the study, acquisition and analysis of data and interpretation of results, writing and critical review of the manuscript, gave approval of the final version of the manuscript to be published, and agreed to be accountable for the integrity of the data and the accuracy of the work. All authors read and approved the final manuscript.

\section{Ethics approval and consent to participate}

This study was performed in accordance with the AMCH Institutional Review Board. Patient consent to participate was waived.

\section{Consent for publication}

Not applicable.

\section{Competing interests}

T.P.L. is a consultant for Cubist. M.H.S. has served on a consulting board for Trius Pharmaceuticals with all proceeds being donated to Midwestern University. He is a grant-funded investigator for Cubist Pharmaceutical for an antimicrobial stewardship study. All other authors: none to declare.

\section{Publisher's Note}

Springer Nature remains neutral with regard to jurisdictional claims in published maps and institutional affiliations.

\section{Author details}

'Albany College of Pharmacy and Health Sciences, Albany, NY 12208-3492, USA. ${ }^{2}$ Midwestern University Chicago College of Pharmacy, Downers Grove, IL, USA. ${ }^{3}$ Northwestern Medicine, Chicago, IL, USA. ${ }^{4}$ Harvard School of Public Health, Boston, MA, USA. ${ }^{5} \mathrm{JMI}$ Laboratories, North Liberty, IA, USA. ${ }^{6}$ University at Albany, State University of New York, Albany, NY, USA.

Received: 8 February 2017 Accepted: 18 July 2017

Published online: 02 August 2017

\section{References}

1. Liu C, Bayer A, Cosgrove SE, et al. Clinical practice guidelines by the infectious diseases society of america for the treatment of methicillinresistant Staphylococcus Aureus infections in adults and children: executive summary. Clin Infect Dis. 2011;52(3):285-92.

2. Wootton M, Howe RA, Hillman R, Walsh TR, Bennett PM, MacGowan AP. A modified population analysis profile (PAP) method to detect heteroresistance to vancomycin in Staphylococcus Aureus in a UK hospital. J Antimicrob Chemother. 2001;47(4):399-403.

3. Charles PG, Ward PB, Johnson PD, Howden BP, Grayson ML. Clinical features associated with bacteremia due to heterogeneous vancomycinintermediate Staphylococcus Aureus. Clin Infect Dis. 2004;38:448-51.

4. Casapao AM, Leonard SN, Davis SL, Lodise TP, Patel N, Goff DA, et al. Clinical outcomes in patients with heterogeneous vancomycin-intermediate Staphylococcus Aureus (hVISA) bloodstream infection. Antimicrob Agents Chemother. 2013;57:4252-9.

5. Garner JS, Jarvis WR, Emori $T G$, Horan TC, Hughes JM. CDC definitions for nosocomial infections, 1988. Am J Infect Control. 1988;16:128-40.

6. Wootton M, MacGowan AP, Walsh TR, Howe RA. A multicenter study evaluating the current strategies for isolating Staphylococcus Aureus strains with reduced susceptibility to glycopeptides. J Clin Microbiol. 2007:45:32932.

7. Lodise TP, Drusano GL, Zasowski E, Dihmess A, Lazariu V, Cosler L, et al. Vancomycin exposure in patients with Methicillin-resistant Staphylococcus Aureus bloodstream infections: how much is enough? Clin Infect Dis. 2014; 59:666-75.

8. Clinical and Laboratory Standards Institute. Methods for Dilution Antimicrobial Susceptibility Tests for Bacteria That Grow Aerobically; Approved Standard—Ninth Edition M07-A9. Wayne: CLSI; 2012.

9. D'Argenio DZ, Schumitzky A, Wang X. ADAPT 5 User's guide: pharmacokinetic/Pharmacodynamic systems analysis software. Los Angeles: Biomedical Simulations Resource; 2009.

10. Neely MN, Youn G, Jones B, Jelliffe RW, Drusano GL, Rodvold KA, et al. Are vancomycin trough concentrations adequate for optimal dosing? Antimicrob Agents Chemother. 2014:58:309-16.

11. Craig WA. Basic pharmacodynamics of antibacterials with clinical applications to the use of beta-lactams, glycopeptides, and linezolid. Infect Dis Clin N Am. 2003;17:479-501. 
12. Craig WA, Andes DA. Abstracts of the forty-sixth annual meeting of the Interscience conference on antimicrobial agents and chemotherapy, San Francisco, CA, 2006. Abstract A-644. In: In vivo pharmacodynamics of vancomycin against VISA, heteroresistant VISA (hVISA) and VSSA in the neutropenic murine thigh-infection model. Washington: American Society for Microbiology.

13. van Hal SJ, Paterson DL. Systematic review and meta-analysis of the significance of heterogeneous vancomycin-intermediate Staphylococcus Aureus isolates. Antimicrob Agents Chemother. 2011;55:405-10.

14. Centers for Disease Control and Prevention. Antibiotic Resistance Threats in the United States. Department of Health and Human Services; 2013. http:// www.cdc.gov/drugresistance/threat-report-2013/pdf/ar-threats-2013-508.pdf. Accessed 6 Feb 2017

Submit your next manuscript to BioMed Central and we will help you at every step:

- We accept pre-submission inquiries

- Our selector tool helps you to find the most relevant journal

- We provide round the clock customer support

- Convenient online submission

- Thorough peer review

- Inclusion in PubMed and all major indexing services

- Maximum visibility for your research

Submit your manuscript at www.biomedcentral.com/submit
Biomed Central 